\title{
IMPACTO VOCAL DE PROFESSORES
}

\section{Teachers' vocal impact}

Adriana Ricarte (1), Silvana Bommarito (2), Brasília Chiari ${ }^{(3)}$

\begin{abstract}
RESUMO
Objetivo: analisar o impacto vocal nas atividades diárias em professores do ensino médio. Correlacionar os achado da auto-percepção do problema vocal com os aspectos: efeitos no trabaIho, na comunicação diária, na comunicação social e na sua emoção. Método: a amostra foi constituída por 107 professores, sendo $86 \mathrm{com}$ queixa e $21 \mathrm{sem}$ queixa, selecionados em escolas da rede particular de ensino de Maceió-AL. Cada professor respondeu individualmente o protocolo Perfil Participação em Atividades Vocais na presença da pesquisadora, assinalando suas respostas em uma escala visual que varia de 0 a 10. O protocolo é composto por 28 questões com a presença integrada em cinco aspectos englobados para avaliar a qualidade de vida e o resultado de tratamentos vocais. O protocolo oferece, ainda, dois escores adicionais: pontuação de limitação nas atividades (PLA) e de restrição de participação (PRP). Resultados: na comparação dos grupos com e sem queixa vocal foram verificados que todos os resultados foram estatisticamente significantes $(p<0,001)$ e, em todos os aspectos os maiores escores foram do grupo com queixa vocal. A PLA foi de 33,27, em um total de 100 pontos e a PRP foi de 20,46, em um total de 100 pontos e a pontuação da média dos aspectos foi de 79,50, em um total de 280 pontos. Conclusão: a média dos aspectos analisados foi de 79,50 pontos verificando-se que os professores se sentem limitados no exercício da sua profissão, porém não restritos a exercê-la. Quanto à correlação dos aspectos todos foram estatisticamente significantes, demonstrando fidedignidade. Os escores adicionais PLA e PRP apresentaram valores que reforçam a limitação em exercer a docência.
\end{abstract}

DESCRITORES: Voz; Qualidade de Vida; Docentes; Atividades Cotidianas

\section{INTRODUÇÃO}

A Organização Mundial de Saúde (OMS) refere o termo saúde como um conceito multidimensional que engloba aspectos físicos, mentais e sociais,

(1) Fonoaudióloga Clínica do centro avançado de otorrinolaringologia e cirurgia estética facial - SINUS; Especialista em Voz pelo CFFa; . Maceió, AL, Brasil; Mestranda em Distúrbios da Comunicação Humana pela Universidade Federal de São Paulo - Escola Paulista de Medicina - UNIFESP/ EPM.

(2) Fonoaudióloga Clínica; Professora Adjunto do Departamento de Fonoaudiologia da Universidade Federal de São Paulo UNIFESP/EPM; São Paulo, SP, Brasil; Doutora em Distúrbios da Comunicação Humana pela Universidade Federal de São Paulo - Escola Paulista de Medicina UNIFESP/EPM.

(3) Fonoaudióloga; Professora Livre-docente do Departamento de Fonoaudiologia da Universidade Federal de São Paulo - Escola Paulista de Medicina - UNIFESP/EPM. São Paulo - SP. Brasil.

Conflito de interesses: inexistente não se limitando apenas à ausência de doença1. Recentemente esse conceito foi ampliado, incluindo a qualidade de vida, que é definida como sendo a percepção do indivíduo de sua posição na vida, relevando-se os objetivos, expectativas, padrões sócio-culturais-econômicos e interesses pessoais, pois enfocam o bem-estar do indivíduo².

A mensuração do bem-estar e da qualidade de vida é a base da avaliação de resultados de tratamento e sua sustentação. Após a crise da saúde em 1969 nos Estados Unidos (EUA) os pacientes passaram a ter dúvidas sobre a eficácia dos procedimentos médicos, verificando-se que o problema estava na inabilidade de medir e entender os efeitos das escolhas de acordo com as aspirações do paciente por uma melhor qualidade de vida. A produção e validação de instrumentos de autoavaliação da qualidade de vida, é necessária para que os resultados do tratamento e seus impactos na vida do paciente sejam avaliados ${ }^{3}$. 
Dessa maneira os instrumentos que avaliam resultados de tratamento e/ou qualidade de vida devem ser rigorosamente elaborados a partir de declarações de pacientes com diferentes doenças, pessoas saudáveis e profissionais da saúde. A partir de então esses instrumentos devem ser submetidos às medidas psicométricas de validade, confiabilidade e sensibilidade, para que sejam comprovados e, posteriormente, utilizados.

Avaliar a voz se faz necessário, uma vez que está presente nos processos de socialização humana, como um dos componentes da linguagem oral e de relação interpessoal, produzindo impactos na qualidade de vida dos indivíduos, especialmente daqueles que fazem o uso da voz falada e/ou cantada em sua profissão4,5.

Na profissão docente, a voz é fator relevante para o desempenho profissional e a atuação do professor em sala de aula, especialmente enquanto componente constitutivo da identidade do professor como trabalhador, do impacto do docente sobre o discente e do componente do processo ensinoaprendizagem ${ }^{5}$. Em contrapartida, o uso inadequado da voz pode gerar uma disfonia que é um transtorno da voz, que pode ter três graus de acometimento: discreto, moderado e severo ${ }^{9}$. Tradicionalmente as avaliações objetivas de uma disfonia podem apresentar resultados dentro dos limites da normalidade, enquanto protocolos de qualidade de vida e análises subjetivas oferecem diversas informações sobre o impacto da dificuldade da produção vocal, o que pode contribuir enormemente para a compreensão do o que o paciente de fato sente em decorrência da disfonia.

A voz do professor assume características e modulações diferentes de acordo com os mais diversos objetivos que quer atingir, sendo norteada pela reação dos alunos. A voz é um recurso poderoso para obtenção da atenção do aluno em sala de aula, contribuindo para envolvê-lo na produção do conhecimento $^{10}$.

No Brasil atualmente existem três protocolos válidos para analisar a qualidade de vida incorporando aspectos vocais, Qualidade de Vida e Voz - QVV6, que é um instrumento composto por 10 itens desenvolvido especificamente para avaliar a qualidade de vida e o resultado de tratamentos em transtornos vocais, contendo dois domínios: um físico e um sócio-emocional. Perfil de Participação e Atividades Vocais - PPAV7, é um instrumento composto por 28 questões, com a presença integrada em cinco aspectos desenvolvidos especificamente para avaliar a qualidade de vida e o resultado de tratamentos em transtornos vocais e o protocolo Índice de Desvantagem Vocal - IDV ${ }^{8}$, que é um instrumento composto de 30 questões, caracterizado pela presença de três domínios: emocional, funcional e orgânica.

O protocolo contemplado na pesquisa foi o PPAV, por ser um protocolo de rápida e fácil aplicação e que disponibiliza dados importantes sobre o impacto da voz na vida dos sujeitos, e por ser o único que apresenta dois escores adicionais que o PLA (pontuação de limitação nas atividades) e o PRP (pontuação de restrição de participação), que abordam a diferença entre a limitação promovida pelo problema vocal e a disposição do indivíduo em participar de atividades de vida diária.

A partir dessas premissas objetivou-se analisar o impacto vocal nas atividades do dia a dia de professores do ensino médio e correlacionar os achados da auto-percepção da severidade do seu problema vocal com os achados nos demais aspectos (efeitos no trabalho, efeitos na comunicação diária, efeitos na comunicação social e efeitos na emoção). Assim, a questão relevante a responder nesta pesquisa é: quais os efeitos dos impactos vocais em atividades do dia a dia de professores do ensino médio?

\section{MÉTODO}

O projeto desta pesquisa foi submetido para apreciação dos Comitês de Ética em Pesquisa da Universidade Federal de São Paulo (UNIFESP) / Hospital São Paulo, São Paulo, SP; Processo no 1620/08 e da Universidade Estadual de Ciências da Saúde de Alagoas (UNCISAL), Maceió, AL; Processo no 765 , sendo a coleta de dados iniciada apenas após sua aprovação. Todos os participantes concordaram em participar do estudo e assinaram o Termo de Consentimento Livre e Esclarecido. Cabe ressaltar que o tipo de estudo desta pesquisa foi o transversal descritivo.

O protocolo foi aplicado em 107 professores, sendo 86 que formaram o grupo com queixa vocal e 21 formaram o grupo sem queixa vocal. A idade dos participantes variou de 24 até 64 anos, com média de 37,2 anos no grupo com queixa e de 35,6 anos no grupo sem queixa vocal. Do total de professores, $70(64,4 \%)$ eram do sexo masculino, sendo 84,2 no grupo com queixa vocal e $15,8 \%$ no grupo sem queixa vocal, e $37(34,6 \%)$ do sexo feminino, sendo $72,9 \%$ no grupo com queixa vocal e $27,1 \%$ no grupo sem queixa vocal.

A amostra foi obtida por meio probabilístico por conglomerado para a seleção das escolas e uma amostra aleatória simples para a seleção dos professores, sem reposição, proporcional à quantidade de professores por escola. As escolas foram enumeradas de 1 até 83 , e foi realizado um sorteio que contemplou 7 escolas, e a partir de então foram 
sorteados de maneira aleatória aproximadamente 15 professores de cada escola.

Para selecionar a amostra foram utilizados como critério de inclusão os professores de ensino médio da rede particular cuja condição de professor pudesse ser comprovada pela apresentação de um documento de identificação que tivesse a foto do professor, por exemplo, crachá de identificação no momento do recrutamento do professor para participar da pesquisa. Foram excluídos da pesquisa os professores da rede estadual ou municipal e os que realizavam ou haviam realizado fonoterapia com o intuito de uniformizar a amostra.

Os indivíduos selecionados foram convidados a participar da pesquisa pelo próprio pesquisador principal. Neste momento, foram apresentadas informações sobre a pesquisa como (objetivos, riscos, benefícios, e procedimentos aos quais serão submetidos). Confirmado o desejo de participar voluntariamente da pesquisa, foi entregue uma cópia do Termo de Consentimento Livre e Esclarecido para que o mesmo o assinasse.

Inicialmente foi aplicada uma breve anamnese composta por dados de identificação pessoal como nome, sexo e idade.

Posteriormente foi aplicado um questionário padronizado Perfil de Participação e Atividades Vocais (Figura 1), aplicado individualmente pela própria pesquisadora em cada professor apenas uma vez. Apenas na resposta da escala visual cada pesquisado deveria assinalar com uma caneta o grau de severidade de seu problema. O protocolo utilizado foi o PPAV validado no Brasil (Ricarte, Behlau, Oliveira 2006), instrumento composto por 28 questões, com a presença integrada em cinco aspectos desenvolvidos especificamente para avaliar a qualidade de vida e o resultado de tratamentos em transtornos vocais, considerando-se:

Auto-percepção do grau de seu problema vocal: refere-se ao primeiro aspecto abordado no questionário, formado por apenas uma pergunta, com a pontuação máxima de 10 pontos, representa o que o sujeito pensa sobre sua própria voz.

Efeitos no trabalho: é o segundo aspecto formado por quatro perguntas, com a pontuação máxima de 40 pontos, que contemplam achados sobre o exercício profissional atual e nos seis últimos meses de atuação.

Efeitos na comunicação diária: compõem o terceiro aspecto do protocolo, formado por doze perguntas, com a pontuação máxima de 120 pontos, que questionam o indivíduo, atualmente e nos últimos seis meses, sobre uso do telefone, comunicação em ambientes ruidosos e silenciosos e à transmissão da mensagem.
Efeitos na comunicação social: é o quarto aspecto do protocolo, formado por quatro perguntas, com a pontuação máxima de 40 pontos, referentes ao prejuízo vocal em atividades sociais, atualmente e nos últimos seis meses. Duas perguntas são gerais e duas sobre a percepção da família, amigos ou colegas de trabalho sobre o problema de voz do professor entrevistado.

Efeitos na sua emoção: compõe o último aspecto do protocolo, é formado por sete perguntas, com a pontuação máxima de 70 pontos, que elencam características emocionais como vergonha, auto-estima, chateação, preocupação, insatisfação, personalidade e auto-imagem.

O protocolo apresenta dois escores adicionais chamados de Pontuação de Limitação nas Atividades (PLA) que é composto por dez perguntas em três diferentes domínios, que elencam a impossibilidade da realização de atividades, em aspectos profissionais e de comunicação, e a Pontuação de Restrição de Participação (PRP), escore também composto por dez perguntas em três distintos domínios, que reforçam a restrição que o indivíduo sente ao realizar as atividades em aspectos profissionais e de comunicação.

Os escores, dos aspectos e do valor total, foram calculados por uma fórmula padrão e poderiam variar de 0 a 10 , sendo que 0 indica normalidade e 10 severidade. Para cada resposta, um traço na vertical foi assinalado em uma escala analógica de $10 \mathrm{~cm}$ e o valor assinalado foi medido com régua. Para cada questão a pontuação podia variar de zero a dez.

A partir do preenchimento dos escores, os valores máximos encontrados são: no primeiro aspecto (auto-percepção do grau de seu problema vocal) a pontuação máxima dez, no segundo (efeitos no trabalho) quarenta, no terceiro (efeitos da comunicação diária) cento e vinte pontos, no quarto (efeitos na comunicação social) quarenta pontos e no quinto (efeitos na sua emoção) setenta pontos, o que pode somar um resultado máximo de 280 pontos. Os escores adicionais PLA e PRP foram calculados respectivamente pela soma das questões 2, 4, 6, 8, 10,12, 14, 16, 18 e 20, e a soma questões $3,5,7,9,11,13,15,17,19$ e 21, totalizando no máximo 100 pontos em cada escore.

\section{Metodologia Estatística}

O objetivo deste estudo foi avaliar o impacto vocal nas atividades do dia a dia de professores, por meio do protocolo Perfil de Participação e Atividades Vocais. Neste protocolo consta um total de 28 perguntas, cada qual sendo medida por meio de uma escala analógica visual de $10 \mathrm{~cm}$, onde o professor assinala com uma 


\section{PROTOCOLO DO PERFIL DE PARTICIPAÇÃO E ATIVIDADES VOCAIS - PPAV}

\section{Auto-percepção do grau de seu problema vocal}

$1 . \quad$ O quanto o seu problema de voz é intenso? Normal |

| Intenso

\section{Efeitos no trabalho}

2. Seu trabalho é afetado pelo seu problema de voz?

Normal |__ Severo

3. Nos últimos 6 meses você chegou a pensar em mudar seu trabalho por causa do seu problema de voz?

Normal |__ Severo

4. Seu problema de voz criou alguma pressão em seu trabalho?

Normal |__ | Severo

5. Nos últimos 6 meses, o seu problema de voz tem afetado o futuro de sua carreira profissional? Normal |__ Severo

\section{Efeitos na comunicação diária}

6. As pessoas pedem para você repetir o que acabou de dizer por causa do seu problema de voz?

Normal |__ | Severo

7. Nos últimos 6 meses você alguma vez evitou falar com as pessoas por causa do seu problema de voz?

Normal |__ Severo

8. As pessoas têm dificuldade de compreender você ao telefone por causa do seu problema de voz?

Normal |__ | Severo

9. Nos últimos 6 meses você reduziu o uso do telefone por causa do seu problema de voz?

Normal |__ Severo

10. O seu problema de voz afeta sua comunicação em ambientes silenciosos?

Normal |__ Severo

11. Nos últimos 6 meses você chegou a evitar conversas em ambientes silenciosos por causa do seu problema de voz?

Normal |__ Severo

12. O seu problema de voz afeta sua comunicação em ambientes ruidosos?

Normal |__ Severo

13. Nos últimos 6 meses você alguma vez chegou a evitar conversas em ambientes ruidosos por causa do seu problema de voz?

Normal |_ Severo

14. Seu problema de voz afeta sua mensagem quando você está falando para um grupo de pessoas?

Normal |__ Severo

15. Nos últimos 6 meses você alguma vez evitou conversas em grupo por causa do seu problema de voz?

Normal | | Severo

16. O seu problema de voz afeta na transmissão da sua mensagem?

Normal |_ Severo

17. Nos últimos 6 meses você alguma vez evitou falar por causa do seu problema de voz?

Normal | Severo

\section{Efeitos na comunicação social}

18. Seu problema de voz afeta suas atividades sociais?

Normal | _ Severo

19. Nos últimos 6 meses você evitou atividades sociais por causa do seu problema de voz?

Normal |_ | Severo

20. Sua família, amigos ou colegas de trabalho se incomodam com seu problema de voz?

Normal |__ Severo

21. Nos últimos 6 meses alguma vez você evitou comunicar-se com seus familiares, amigos ou colegas de trabalho por causa do seu problema de voz?

Normal |__ Severo

\section{Efeitos na sua emoção}

22. Você se sente chateado por causa do seu problema de voz?

Normal |__ | Severo

23. Você está envergonhado pelo seu problema de voz?

Normal |___ Severo

24. Você está com baixa auto-estima por causa do seu problema de voz?

Normal |___ Severo

25. Você está preocupado por causa do seu problema de voz?

Normal |__ | Severo

26. Você se sente insatisfeito por causa da sua voz?

Normal |__ | Severo

27. Seu problema de voz afeta sua personalidade?

Normal |__ Severo

28. Seu problema de voz afeta sua auto-imagem?

Normal 
caneta qual a importância daquele assunto na sua atividade diária.

Quanto maior a marcação na escala, maior a percepção do problema vocal.

Foram incluídos professores com e sem queixa vocal e os resultados comparados entre esses dois grupos.

Inicialmente os dados foram resumidos por meio de algumas estatísticas, tais como média, desvio padrão (dP), valor mínimo, máximo e mediana para as variáveis numéricas, e por meio da frequência absoluta $(\mathrm{N})$ e relativa (\%) no caso de variáveis categóricas.

A comparação entre os dados numéricos foi obtida pelo teste t para amostras independentes, e pelo teste de Qui-quadrado no caso de variáveis categóricas.

Cada pergunta do protocolo Perfil de Participação e Atividades Vocais foi analisada separadamente, comparando-se sempre os grupos com e sem queixa vocal.
Somente para o grupo de professores com queixa vocal, as questões específicas de um mesmo aspecto foram comparadas entre si, de forma a se verificar, dentro daquele aspecto, quais as questões que mais contribuíram para o problema vocal. Neste caso foi utilizado a Análise de Variância, e em caso de significância estatística, foi utilizado também o teste de comparações múltiplas de Tukey para saber exatamente quais questões alcançaram pontuação diferente entre si do ponto de vista estatístico.

Além dessas análises, ainda no grupo de professores com queixa vocal, também foi avaliado o grau de associação entre os diversos aspectos do protocolo Perfil de Participação e Atividades Vocais, medido por meio da Correlação de Pearson.

Todas as análises foram obtidos com a utilização do software estatístico Minitab, versão 15.1. Significância estatística foi considerada para valores de $p<0,05$.

\section{RESULTADOS}

Tabela 1 - Distribuição das medidas referentes à pontuação total do protocolo PPAV de professores, com e sem queixa vocal

\begin{tabular}{lccccccc}
\hline Pergunta & $\mathbf{N}$ & Média & d.p. & mínimo & mediana & Máximo & p \\
\hline Somatória total (28 perguntas) & do protocolo PPAV \\
c/ queixa & 86 & 79,5 & 46,1 & 11,0 & 67,5 & 222,8 & $<0,001$ \\
s/ queixa & 21 & 9,5 & 7,0 & 0,3 & 7,0 & 24,7 & \\
\hline \multicolumn{10}{l}{ d.p. = desvio padrão } \\
p = nível de significância do teste t, para amostra independentes
\end{tabular}

Verifica-se nos valores representados que a comparação entre os grupos com e sem queixa vocal foi estatisticamente significante $(p<0,001)$, indicando que a percepção da severidade do problema de voz é maior no grupo com queixa vocal (5,56 pontos de um total previsto de 10 pontos).

Tabela 2 - Distribuição das medidas da somatória das respostas P2 + P6 + P18: limitação de pontuação das atividades vocais de professores com e sem queixa vocal

\begin{tabular}{|c|c|c|c|c|c|c|c|}
\hline Pergunta & $\mathbf{N}$ & Média & d.p. & mínimo & mediana & Máximo & 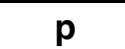 \\
\hline \multicolumn{8}{|c|}{ Limitação de atividades (P2+P6+P18) } \\
\hline c/ queixa & 86 & 11,1 & 6,4 & 0,8 & 9,8 & 28,9 & $<0,001$ \\
\hline s/ queixa & 21 & 1,2 & 1,1 & 0,0 & 0,7 & 4,5 & \\
\hline
\end{tabular}

Observa-se nos valores representados que a comparação entre os grupos com e sem queixa vocal em relação ao escore pontuação de limitação de atividades foi estatisticamente significante $(p<0,001)$, indicando que o valor é maior no grupo com queixa vocal $(33,27$ pontos de um total previsto de 100). 
Tabela 3 - Distribuição das medidas da somatória das respostas das questões P3 + P7 + P19: restrição de participação das atividades de professores com e sem queixa vocal

\begin{tabular}{|c|c|c|c|c|c|c|c|}
\hline Pergunta & $\mathbf{N}$ & Média & d.p. & mínimo & mediana & Máximo & $\bar{p}$ \\
\hline \multicolumn{8}{|c|}{ Limitação de atividades (P3+P7+P19) } \\
\hline c/ queixa & 86 & 5,5 & 4,8 & 0,2 & 4,1 & 25,0 & $<0,001$ \\
\hline s/ queixa & 21 & 0,8 & 0,8 & 0,0 & 0,5 & 2,6 & \\
\hline
\end{tabular}

d.p. = desvio padrão

$\mathrm{p}=$ nível de significância do teste $\mathrm{t}$, para amostras independentes

Constata-se nos valores representados que a comparação entre os grupos com e sem queixa vocal em relação ao escore pontuação de limitação de atividades foi estatisticamente significante
( $p<0,001)$, indicando que o valor é maior no grupo com queixa vocal $(20,46$ pontos de um total previsto de 100).

Tabela 4 - Correlação entre os diferentes aspectos do protocolo de Perfil de Participação e Atividades Vocais, para os sujeitos com queixa vocal

\begin{tabular}{lcccc}
\hline Aspecto & $\begin{array}{c}\text { 1. auto- } \\
\text { percepção }\end{array}$ & 2. trabalho & $\begin{array}{c}\text { 3. comunicação } \\
\text { diária }\end{array}$ & $\begin{array}{c}\text { 4. comunicação } \\
\text { social }\end{array}$ \\
\hline 2. trabalho & $\begin{array}{c}r=0,583 \\
(p<0,001)\end{array}$ & - & - & - \\
3. com. diária & $r=0,592$ & $r=0,576$ & & - \\
4. com. social & $(p<0,001)$ & $(p<0,001)$ & - & - \\
5. emoção & $r=0,470$ & $r=0,380$ & $r=0,721$ & - \\
& $(p<0,001)$ & $(p<0,001)$ & $(p<0,001)$ & $r=0,702$ \\
\hline
\end{tabular}

$r=$ correlação de Pearson

$\mathrm{p}=$ nível de significância do teste para a correlação

A tabela 4 mostra que, para os participantes com queixa vocal, todos os aspectos analisados no protocolo PPAV apresentaram correlação estatisticamente significante entre si $(p<0,001)$, ou seja, quanto maior o grau de importância de determinado aspecto, maior o grau de importância no outro aspecto.

\section{DISCUSSÃO}

O professor é um dos profissionais que mais recorrem aos consultórios de fonoaudiologia devido à grande ocorrência de disfonia ${ }^{11}$. Isto se deve, na maioria das vezes, à falta de preparo e informação sobre produção vocal e cuidados para preservar a saúde da voz, associados às más condições físicas e sociais (estrutura física da escola e relação com alunos e empregadores).

$\mathrm{Na}$ formação de um professor, geralmente não constam informações sobre a saúde da voz e, quando ocorrem, são transmitidas por meio de palestras e atendimentos individualizados, já com o profissional no mercado de trabalho ${ }^{9}$. Caso isso ocorresse na formação acadêmica, quando ele começasse a lecionar estaria mais alerta aos problemas vocais, podendo possuir uma maior percepção em relação à voz e provavelmente tomaria os cuidados necessários para evitar problemas futuros. A literatura ainda afirma que poucos professores cultivam o hábito de procurar ajuda profissional preventiva ${ }^{12,13}$.

Conforme referido no método, foi utilizado para realização dessa pesquisa o protocolo perfil de participação e atividades vocais (PPAV) que é um protocolo validado ${ }^{8}$ foi escolhido por ser de fácil compreensão, com simples aplicação e sensível à influência da disfonia na qualidade de vida, apresentando dois escores adicionais o PLA (pontuação de limitação da atividade) e o PRP (pontuação de restrição de participação). A literatura afirma que a base da avaliação de resultados de tratamentos e sua sustentação deve ocorrer pela mensuração do bem-estar e da qualidade de vida ${ }^{3}$.Analisando-se os 
cinco aspectos do protocolo, o valor médio encontrado foi de 79,50 pontos no grupo com queixa vocal e de 9,50 pontos no grupo sem queixa vocal em uma escala de zero a duzentos e oitenta pontos (tabela 1). Os valores encontrados foram estatisticamente significantes, havendo pontuação reduzida nos participantes do grupo controle, ou seja, sem queixa vocal. Em pesquisas com o mesmo protocolo os valores encontrados foram coerentes com o tipo de população estudada ${ }^{7,14}$. Concordando com a literatura encontrada é fato afirmar que a restrição de participação e atividades vocais não se evidenciou na população estudada, demonstrando que há necessidade de atuação com essa população sobre alterações vocais e tratamento.

Com relação aos escores da pontuação de limitação nas atividades (PLA) e pontuação de restrição de participação (PRP), os valores médios encontrados foram de 33,27 pontos e 20,46 pontos no grupo com queixa vocal e de 3,86 e 2,97 pontos no grupo sem queixa vocal, respectivamente, em uma escala de zero a cem pontos cada, respectivamente, em uma escala de zero a trinta pontos cada, respectivamente (tabelas 2 e 3). Estes resultados demonstram que os professores com queixa vocal se sentem por vezes limitados em exercer suas funções de docente e as demais do dia a dia. Nossos achados são semelhantes aos descritos na literatura mencionados ${ }^{15}$. Outros autores que também utilizaram o PPAV (Perfil de Participação e Atividades Vocais) e encontraram limitação nos professores pesquisados em exercer sua função de docente ${ }^{15,16}$

$\mathrm{Na}$ tabela 4, após aplicação do teste de coeficiente de correlação de Pearson, pode ser encontrado o valor de $p$, adotando a esse valor como $p<1 \%$, assim pode ser verificado que os valores são considerados estatisticamente significantes na correlação dos diferentes aspectos do protocolo, para os sujeitos com queixa vocal. $\mathrm{Na}$ literatura o valor de significância, concordando com o utilizado na pesquisa foi de $p<1 \%$, para que o valor encontrado tenha um respaldo estatístico mais significante ${ }^{17}$.

Os protocolos de qualidade de vida que abrangem voz são um excelente método para identificar alterações e mensurar o tratamento, de acordo com a percepção do indivíduo, captando o que essa alteração pode causar e como o indivíduo pode se sentir limitado a exercer sua profissão, tornando assim, a conduta médica e o tratamento clínico mais rápido e eficiente ${ }^{18,19}$.
A literatura apresenta um estudo com a validação dos três protocolos relacionados à qualidade de vida e voz existentes no Brasil, sugerindo o uso desses protocolos para avaliação vocal, bem como para análise de resultados de tratamento ${ }^{20}$.

O resultado da pesquisa demonstrou que a maioria da população pesquisada se sente acometida por um problema vocal, muitos referem até haver limitação em exercer atividades do dia a dia e sua atividade profissional, porém a minoria pensa em mudar de carreira. Outro estudo afirma que os sintomas vocais devem ser valorizados em programas de saúde vocal, para que haja identificação prévia por parte dos professores e tratamento preventivo, caso necessário ${ }^{21,22}$.

Há necessidade de um esclarecimento prévio, antes da aplicação de uma nova pesquisa, do que são alterações vocais e o que elas podem acarretar se não forem devidamente tratadas, para que haja maior compreensão por parte da população pesquisada.

Diante dos resultados encontrados, é importante avaliar a mesma população depois da realização de terapia vocal, observando as prováveis alterações nos escores do protocolo PPAV. Outra sugestão é fazer a mesma pesquisa com os professores de escola pública, que são funcionários públicos, que trabalham com a segurança de um emprego fixo, com más condições de ambiente de trabalho, para que haja uma nova pesquisa que diferencie as duas populações utilizando o mesmo protocolo.

\section{CONCLUSÃO}

A média dos aspectos analisados foi de 79,50 pontos, verificando-se assim que os professores com queixa vocal se sentem limitados em exercer sua profissão, porém não restritos a exercê-la. Correlacionando a qualidade de vida, observa-se que o impacto da voz na vida dos professores ainda é pouco percebido pelos mesmos.

Quanto à correlação da auto-percepção do problema de voz com os demais aspectos do protocolo, pode ser verificado que todos os dados analisados foram estatisticamente significantes, o que demonstra a fidedignidade do estudo, já que foi adotado um valor de significância $p<1 \%$.

Os escores adicionais de limitação e restrição de participação nas atividades vocais do dia a dia apresentaram valores de 33,27 e 20,46 pontos respectivamente, reforçando que os professores com queixa vocal, de fato, se sentem limitados em exercer sua função de docente. 


\begin{abstract}
Purpose: to analyze the vocal impact in the daily activities on high-school teachers. Correlate the finding of the auto-perception on the vocal problem with the following aspects: effects in the work, daily communication, social communication and, its emotion Method: the sample consisted of 107 teachers, 86 with and 21 with no complaint, selected from private teaching schools in Maceió-AL. Each teacher answered individually the Protocol for Voice Activity Participation Profile in the presence of the researcher, noting their responses on a visual scale ranging from 0 to 10 . The protocol is composed of 28 questions with the presence integrated in five aspects to evaluate the quality of life and the result of vocal treatments. The protocol offers, still, two additional scores: punctuation of limitation in the activities (PLA) and restriction of participation (PRP). Results: comparing the groups with and with no vocal complaints, the aspects demonstrated that all the results had been statistical significant $(p<0.001)$ and, all the greater scores were related to the group with vocal complaint. The PLA was 33.27, total of 100 points. The PRP was 20.46 , total of 100 points. The punctuation for the average of the aspects was 79.50 , total of 280 points. Conclusion: the average for the analyzed aspects was 79.50 points verifying so that teachers feel limited in the exercise of their profession, but not limited to exercise it. The correlation of the aspects had a statistical significance, demonstrating reliability. The PLA and PRP additional scores delivered values that reinforced the limitation in exerting teaching activity.
\end{abstract}

KEYWORDS: Voice; Quality of Life; Faculty; Activities of Daily Living

\section{REFERÊNCIAS}

1. World Health Organization. Physical. Measuring Quality of Life - The World Health Organization Quality of Life Instruments. WHO/MSA/MNH/PSKF; 1997;1-15.

2. Soarez PC, Kowalski CCG, Ferraz MB, Ciconelli RM. Tradução para português brasileiro e validação de um questionário de avaliação de produtividade. Rev Panam Salud Publica; 2007;22(1)21-8.

3. Ellwood PM. Shattuck Lecture - Outcomes management: a technology of patient experience. $\mathrm{N}$ Engl J Med; 1988;318:1552-3.

4. Benninger S, Ahuja AS, Gardner G, Grywalski C. Assessing outcomes for dysphonic patients. J Voice; 1998;12(4):540-50.

5. Grillo $\mathrm{MH}$, Penteado RZ. Impacto vocal na qualidade de vida de professore(a)s do ensino fundamental. Pró-Fono R. Atual. Cient.; 2005;17(3):321-30.

6. Gasparini G, Behlau M. Quality of Life: Validation of the Brazilian Version of the Voice-Related Quality of Life (V-RQOL) Measure. J Voice 2009;23(1):76-81.

7. Ricarte A, Behlau M, Oliveira G. Validação do protocolo perfil de participação e atividades vocais (PPAV) no Brasil. Anais do XIV Congresso Brasileiro de Fonoaudiologia, 2006.

8. Santos L, Oliveira G, Behlau M. Cross-Cultural adaptation and validation of the voice handicap index brazilian portuguese. J Voice 2011;25(3):354-9.
9. Behlau $M$, Madázio $G$, Feijó $D$, Pontes $P$. Avaliação da voz. In: Behlau M. Voz: o livro do especialista. Rio de Janeiro: Revinter; 2001.

10. Carmo RD, Camargo Z, Nemr K. Relação entre qualidade de vida e auto-percepção da qualidade vocal de pacientes laringectomizados totais: estudo piloto. Rev CEFAC. 2006; 8(4):518-28.

11. Simões M. A Voz do Professor: Histórico da Produção Científica de Fonoaudiólogos Brasileiros sobre o Uso da Voz nessa Categoria Profissional. In: Ferreira LP, Oliveira SMRP. Voz Profissional: Produção Científica da Fonoaudiologia Brasileira, Roca, São Paulo; 2004.

12. Vieira AC, Behlau M. Análise de voz e comunicação oral de professores de curso pré-vestibular. Rev Soc Bras Fonoaudiol. 2009; 14(3):346-51.

13. Bassi IB, Assunção AA, Medeiros AM, Gama ACC, Menezes LN. Análise dos parâmetros vocais, laríngeos e do protocolo do perfil de participação e atividades vocais (PPAV) em professoras da rede municipal de ensino de Belo Horizonte encaminhadas para fonoterapia. Anais do XVI Congresso Brasileiro de Fonoaudiologia, 2008:414.

14. Ferraz PRP, Behlau M, Oliveira G. AutoAvaliação do impacto vocal nas atividades de vida diária de professores por meio do protocolo PPAV. [monografia de especialização] São Paulo: Centro de Estudos da Voz; 2007. 
15. Kasama S, Brassolotto AG. Percepção vocal e qualidade de vida. ProFono Rev Atual Cient. 2007;19(1):19-28.

16. Ma EP-M, Yiu EM-L. Voice activity and participation profile: Assessing the impact of voice disorders on daily activities. J Speech Hear Res; 2001;44:511-24.

17. Silverio KC, Gonçalves CG, Penteado RZ, Vieira TP, Libardi A, Rossi D. Ações em saúde vocal: proposta de melhoria do perfil vocal de professores. ProFono Rev Atual Cient; 2008;20(3):177-82

18. Awan SN, Roy N. Outcomes measurement in voice disorders: aplication of an acoustic index of dysphonia severity. J Speech Hear Res; 2009;52:482-99.
19. Behlau M, Oliveira G, Santos LMA, Ricarte A. Validação no Brasil de protocolos de auto-avaliação do impacto de uma disfonia. Rev Pro-Fono; 2009; 21: 326-32.

20. Penteado ZR. Relações entre saúde e trabalho docente: percepções de professores sobre saúde vocal. Rev Soc Bras Fonoaudiol. 2007;12(1):18-22. 21. Dragone MLS, Behlau M. A fonoaudiologia brasileira e a voz do professor - olhares científicos no decorrer do tempo. Rev Fonoaudiol Brasil; 2006;(4):1-13.

22. Alves LA, Robazzi ML, Marziale HP, Felippe AC, Romano CC. Alterações da saúde e a voz do professor, uma questão de saúde do trabalhador. Rev Latino-Am. Enfermagem; 2009;17(4): 566-72.

Endereço para correspondência:

Adriana Ricarte

Rua Hélio Pradines, no 737 - apto 602

Ponta Verde - Maceió - AL

CEP: 57035-260

E-mail: adriana_ricarte@ hotmail.com 